\title{
Estimulación de la Inteligencia Emocional en mayores: El programa PECI-PM
}

\author{
$\mathrm{M}^{\mathrm{a}}$ del Carmen Pérez-Fuentes, $\mathrm{M}^{\mathrm{a}}$ del Mar Molero, José J. \\ Gázquez y Francisco J. Soler \\ Universidad de Almería (España)
}

\begin{abstract}
El programa de Estimulación Cognitiva e Inteligencia Emocional para personas mayores (PECI-PM), combina técnicas de estimulación cognitiva con la intervención y estimulación sobre aspectos relacionados con la inteligencia emocional. El objetivo del presente trabajo es analizar los resultados obtenidos en inteligencia emocional, tras la primera fase de implementación del PECI-PM. Para ello, se contó con una muestra de 28 usuarios del programa, que habían completado 20 sesiones y participado en las dos evaluaciones (pre-post). La Inteligencia Emocional se evaluó, antes y después de la intervención, mediante el Inventario Breve de Inteligencia Emocional para Mayores (EQ-i-M20). Tras la intervención con el PECI-PM, se observa una mejora, en la mayoría de las dimensiones de inteligencia emocional (Intrapersonal, Interpersonal, Adaptabilidad y Estado de Ánimo). En la dimensión Manejo del Estrés, no se observa un cambio en las puntuaciones obtenidas tras la intervención, lo que lleva a replantear el peso de esta dimensión en la inteligencia emocional de la población mayor. Por otro lado, destaca el papel de la dimensión Interpersonal, que correlaciona significativamente con otros factores de la inteligencia emocional (Intrapersonal y Adaptabilidad), en mayores.
\end{abstract}

Palabras clave: Estimulación cognitiva, Inteligencia emocional, mayores, PECI-PM.

Stimulating emotional intelligence in seniors: The PECI-PM program. The Cognitive Stimulation and Emotional Intelligence for Seniors Program (PECI-PM), combines cognitive stimulation with intervention and stimulation of aspects related to emotional intelligence. The purpose of this study was to analyze the emotional intelligence results of the first stage of PECI-PM implementation. A sample of 28 program users who had completed 20 sessions and participated in the two evaluations (pre-post) was employed for this. Emotional Intelligence was evaluated before and after intervention using the Inventario Breve de Inteligencia Emocional para Mayores [Brief Emotional Intelligence Inventory for Senior Citizens] (EQ-i-M20). After intervention with the PECI-PM, improvement was observed in most of the emotional intelligence dimensions (Intrapersonal, Interpersonal, Adaptability and Mood). In the Stress Management dimension, there was no change in scores after intervention, which leads us to reconsider the weight of this emotional intelligence dimension in the older population. On the other hand, the role of the Interpersonal dimension, which correlates significantly with other emotional intelligence factors (Intrapersonal and Adaptability) in older people, should be emphasized.

Keywords: Cognitive stimulation, Emotional intelligence, seniors, PECI-PM.

Correspondencia: $\mathrm{M}^{\mathrm{a}}$ del Carmen Pérez Fuentes. Facultad de Ciencias de la Educación, Enfermería y Fisioterapia. Universidad de Almería. Ctra. Sacramento, s/n. C.P.: 04120. Almería (España). E-mail: $\underline{\text { mpf421@ual.es }}$ 
Hace más de una década que, la investigación sobre la mejora de las funciones cognitivas en personas mayores, ha concentrado esfuerzos hacia la intervención, tanto en población mayor general (Martin, Clare, Altgassen, Cameron y Zehnder, 2011; Tardif y Simard, 2011), como en personas con deterioro cognitivo (Reijnders, van Heugten y van Boxtel, 2013; Teixeira et al., 2012) o demencia (Coen et al., 2011; Woods, Aguirre, Spector y Orrell, 2012).

Por otro lado, se ha constatado la influencia de las emociones en la toma de decisiones del sujeto y en su conducta (Inglés et al., 2014), partiendo de la idea de una continua interacción entre la emoción y los procesos cognitivos (Eich y Schooler, 2000). Así, desde un enfoque más actual, se hace referencia al estudio de las emociones en el envejecimiento, como otro de los objetivos prioritarios para la mejora de la calidad de vida (Pérez-Fuentes, Ruiz, Molero, Gázquez y Fernández, 2012). Una de las razones que apoyan este planteamiento es la relación que existe entre las emociones y la salud y bienestar de la población mayor (Diener y Chan, 2011; Extremera y Fernández-Berrocal, 2006; Xu y Roberts, 2010).

\section{Emociones y envejecimiento}

A pesar del interés que despierta el estudio de las emociones, Bernarás, Garaigordobil y de las Cuevas (2011), advierten de la escasez de trabajos relacionados con la inteligencia emocional en la vejez. Algunos trabajos han analizado las diferencias evolutivas en IE o las características de la misma en la vejez. Navarro, Latorre y Ros (2009), encontraron diferencias significativas a favor del grupo de mayores, en comparación con adultos jóvenes, en los factores claridad y regulación emocional. Por otro lado, Márquez, Montorio, Izal y Losada (2006), analizaron las diferencias asociadas a la edad en la intensidad emocional y el afrontamiento cognitivo en situaciones estresantes, aunque la mayoría de estos estudios se basan en adolescentes o universitarios (Cabanach, Fariña, Freire, González y Ferradás, 2013). Los autores encontraron diferencias significativas entre jóvenes y mayores, y se apreció una asociación más fuerte de la intensidad emocional y el afrontamiento cognitivo, con respecto a la ansiedad, en el grupo de mayores que en el de jóvenes (Márquez, Montorio, Izal y Losada, 2006). En otros casos (Neupert, Almeida y Charles, 2007), la edad avanzada ha sido relacionada con una menor reactividad emocional a los factores estresantes interpersonales. Más recientemente, Birditt (2013), no encuentra diferencias de edad, en cuanto al tipo de reacciones emocionales negativas experimentadas.

Además, con el paso de los años, la configuración de las relaciones sociales cambia, siendo determinante en la experiencia emocional derivada. Así, los adultos mayores son los que muestran un mayor nivel de experiencias emocionales positivas en la interacción con familiares, mientras que los adultos más jóvenes reportan mayores niveles de afecto positivo en la relación con los amigos (Charles y Piazza, 2007). Las 
personas perciben y organizan el entramado de relaciones sociales, en base a sus respuestas emocionales. En esta línea, Catelli y Lanza (2011), observaron que los adultos mayores eran más propensos que los jóvenes, a apoyarse en la experiencia emocional, en tareas de categorización de estímulos sociales. Según estos resultados, las emociones tendrían un papel destacado en la vida social de la población mayor.

Ante la posibilidad de educar las emociones en la vejez, Scheibe y Zacher (2013), sugieren que, con el paso del tiempo y la ganancia en experiencia, las estrategias y habilidades emocionales se modifican, pudiendo llegar a agudizarse en algunos casos. En esta línea, Bucks, Garner, Tarrant, Bradley y Mogg (2008) observaron que, en la relación con otros, los adultos mayores son capaces de seleccionar las estrategias más eficaces para resolver un problema, y adaptar sus respuestas según la situación. Coats y Blanchard-Fields (2008), al comparar las estrategias de regulación emocional en adultos jóvenes y mayores, encontraron que las diferencias de edad en la regulación emocional ante un problema interpersonal, se explicaban, en parte, por una menor capacidad de los mayores para integrar cognición y emoción.

Orgeta y Phillips (2007) consideran que con la llegada del envejecimiento, resulta más difícil reconocer determinadas emociones negativas, como la tristeza o el miedo. En trabajos más recientes (Suzuki y Akiyama, 2013), se ha comprobado que el deterioro cognitivo asociado a la edad, afecta a la capacidad del sujeto para reconocer emociones. Concretamente, en un grupo de personas mayores, se observó que el déficit relacionado con la edad tenía una influencia negativa en la identificación de emociones como la felicidad, la sorpresa, el miedo y la tristeza.

Por su parte, lejos de las dificultades en el uso de las emociones en la vejez, Kunzmann y Richter (2009), observaron cómo, en determinadas situaciones, los sujetos de edad avanzada experimentan reacciones emocionales equivalentes, o incluso superiores, a la de sujetos más jóvenes. Este fenómeno se conoce como "reactividad emocional", o el nivel en que una persona reacciona a una situación emocional determinada. Estos resultados apoyan la posibilidad de utilizar la inteligencia emocional, para mantener y mejorar la capacidad cognitiva en personas mayores, partiendo de la constatación de un aumento en la actividad cognitiva al usar estímulos con carga emocional.

Para Charles y Carstensen (2010), tanto las relaciones sociales como el bienestar emocional, se benefician de la experiencia. Es decir, la experiencia provee de habilidades de regulación emocional, dando prioridad a los aspectos significativos de la vida. Del mismo modo, según estos autores, a edades avanzadas, los recursos cognitivos también se despliegan de forma selectiva, con la existencia de un sesgo positivo en atención y memoria. 
Inteligencia Emocional y afrontamiento de situaciones estresantes en la vejez

El concepto de estrés tiene una larga trayectoria en el campo de la psicología de la salud, pudiendo enfocar su análisis desde la configuración del medio ambiente, su evaluación, o como respuesta emocional del individuo (Segerstrom y O’Connor, 2012). Así, identificar los factores que moderan los efectos del estrés, se convierte en un gran desafío para la investigación sobre el bienestar de la población mayor (Downing et al., 2012; Rinaldis, Pakenham y Lynch, 2012). Se parte de la idea de que el estrés, y sus formas de afrontamiento, son algo dinámico, a lo largo de las diferentes etapas de la vida. Uno de los factores de estrés más relevantes, con la llegada de la senectud, hace referencia a los cambios de roles en situaciones de crisis, como la jubilación o el duelo (Pearlin, 2010).

Las estrategias de afrontamiento y la inteligencia emocional, han sido analizadas en personas mayores, como un tándem efectivo para moderar los efectos perjudiciales del estrés. Al respecto, autores como Kraaij, Garnefski y Maes (2002), encontraron que los ancianos con mayor autoeficacia de afrontamiento al estrés utilizaban estrategias orientadas a la solución del problema, y una menor carga emocional asociada.

Sobre la forma de afrontar los cambios con la edad, Trouillet, Gana, Lourel y Fort (2009), postulan que el efecto de la edad sobre el afrontamiento al estrés, estaría mediado por otros factores como la autoeficacia percibida y el apoyo social. Para estos autores, la puesta en marcha de determinadas estrategias de afrontamiento al estrés en la senectud, refleja la capacidad del sujeto en los procesos de adaptación al cambio. Por otro lado, como una crítica al uso de estudios transversales para el análisis del afrontamiento con la edad, surgen algunos trabajos de corte longitudinal, como el de Brennan, Holland, Schutte y Moos (2012). Estos autores, observaron durante 20 años, el patrón de afrontamiento al estrés, en un grupo de adultos. Encontraron que, con el paso del tiempo, se daba una disminución general de la capacidad de afrontamiento. No obstante, esta tendencia podría deberse, en gran parte, a la presencia de determinadas variables en la adultez temprana: sintomatología depresiva, escasos recursos económicos, personales y sociales.

Según Scheibe y Carstensen (2010), cuando las personas mayores se enfrentan a acontecimientos negativos inevitables o ineludibles, experimentan niveles relativamente altos de angustia que pueden ser perjudiciales para la salud. Por su parte, Lloyd, Malek-Ahmadi, Barclay, Fernández y Stanley (2012), se preguntaron en qué medida la inteligencia emocional podría predecir la depresión. En una muestra de 128 adultos mayores, aplicaron la Escala de Depresión Geriátrica (GDS) (Yesavage et al., 1983) y el Inventario de Inteligencia Emocional de Bar-On (EQ-i) (Bar-On, 2004). Los resultados mostraron que, por cada punto positivo en el EQ-i, el riesgo de depresión disminuía en un $6 \%$. Por tanto, una mejora en la inteligencia emocional, podría tener un 
efecto beneficioso sobre la evolución de la sintomatología depresiva. Bajo tales circunstancias, se hace necesario contar con herramientas propias de una regulación emocional efectiva.

Así, el objetivo de este trabajo es, no sólo analizar las características de los mayores respecto a la inteligencia emocional, sino también, evaluar los cambios que se producen en los diferentes factores de la inteligencia emocional, tras la intervención con el programa estimulación cognitiva e inteligencia emocional PECI-PM, en un grupo de personas mayores.

\section{MÉTODO}

\section{Participantes}

De los participantes en un taller de estimulación cognitiva e inteligencia emocional $(N=67)$, se seleccionó una muestra de 28 sujetos. Los criterios para la selección de la muestra fueron: haber completado las 20 sesiones de la primera fase del programa y haber cumplimentado la evaluación antes y después de la intervención.

Finalmente, la muestra estuvo formada por un total de 28 usuarios del programa, todos ellos alumnos de la Universidad para Mayores de Almería (España). Con edades comprendidas entre los 60 y los 85 años $(M=69.18 ; D T=6.31)$. Del total de la muestra, el $92.9 \%(N=26)$ eran mujeres, y el 7.1\% $(N=2)$ restante eran hombres.

\section{Instrumentos}

Programa de Estimulación Cognitiva e Inteligencia Emocional para personas mayores (PECI-PM) (Pérez-Fuentes, Molero, Osorio y Mercader, 2014). Se trata de un programa de intervención que combina técnicas de estimulación cognitiva con recursos para el entrenamiento de habilidades propias de la inteligencia emocional, en personas mayores. Su objetivo es trabajar, por un lado, la conservación y mejora del rendimiento en personas mayores, en tareas que requieren la puesta en marcha de las funciones ejecutivas y, por el otro, la mejora y el aprovechamiento de las estrategias en el manejo de las emociones. Consta de diferentes sesiones de juego, adaptando el nivel de dificultad a las características y necesidades del usuario. En cada una de las actividades, el sujeto ha de responder, mediante el uso de una Tablet, a la tarea que se le presenta, manipulando los cubos en la forma que corresponda según el caso. Algunas de las actividades que integran el programa, son: "Ordenar viñetas", "Reconocimiento de emociones", "Intensidad emocional", "Familia de imágenes”, ...

Para la evaluación (pre-post intervención) de la inteligencia emocional, se utilizó la Adaptación para mayores del Emotional Intelligence Inventory: Young Version (EQ-i:YV) de Bar-On y Parker (2000), Inventario de Inteligencia Emocional reducido para mayores (EQ-i-20M), validado y baremado para población mayor española 
(Pérez-Fuentes, Gázquez, Mercader y Molero, 2014). Consta de 20 ítems con cuatro alternativas de respuesta en una escala tipo Likert (1=nunca me pasa, y 4=siempre me pasa) y cinco factores: Intrapersonal, Interpersonal, Manejo del estrés, Adaptabilidad y Estado de ánimo. El valor del Alfa de Cronbach es de .89 para el total de la muestra (.57 Intrapersonal; .80 Interpersonal; .68 Manejo del estrés, .81 Adaptabilidad y .83 Estado de ánimo).

\section{Procedimiento}

La implementación general del programa se lleva a cabo en dos fases: una primera, en la que los participantes completan 20 sesiones (dos veces en semana, con una duración de 45 minutos por sesión). En esta primera fase, el total de los participantes forman parte del grupo experimental. En la segunda fase (sesiones de la 21 a la 40), se incorpora al programa un grupo control, que no ha recibido las 20 primeras sesiones. Los resultados que se presentan en este trabajo, corresponden a los resultados obtenidos en la primera fase de implementación.

La evaluación de la inteligencia emocional se llevó a cabo en dos momentos (Morales, Trianes y Casado, 2013): una evaluación inicial (medida pre-intervención); y, una vez completadas las 20 sesiones, se procede a la segunda evaluación (medida postintervención). En ambos casos, se hace uso de las Tablet, que integran el cuestionario en formato digital.

\section{RESULTADOS}

En la gráfica 1, se presentan las puntuaciones medias obtenidas por el total de muestra, para cada una de las escalas de inteligencia emocional, antes y después de la intervención con el programa (20 sesiones). Tal y como se observa, se produce una ganancia en las puntuaciones medias para la mayoría de las dimensiones analizadas: Intrapersonal (PRE: $M=10.35 ; D T=3.06$; POST: $M=10.56 ; D T=3.11$ ); Interpersonal (PRE: $M=11.29 ; D T=1.75$; POST: $M=12.39 ; D T=1.72$ ); Adaptabilidad (PRE: $M=9.59$; $D T=1.94$; POST: $M=9.76 ; D T=2.13$ ); y Estado de ánimo (PRE: $M=10.80 ; D T=2.61$; POST: $M=10.84 ; D T=2.03)$. El único caso en que no se observa evolución positiva con respecto a la puntuación obtenida en la medida pre-intervención, es en la dimensión Manejo del Estrés (PRE: $M=7.73 ; D T=1.95$; POST: $M=7.23 ; D T=1.29$ ).

A partir de la prueba no paramétrica de Wilcoxon para muestras relacionadas, se constató que las diferencias entre las puntuaciones pre y post, resultaron significativas para la dimensión Interpersonal $(Z=-2.65 ; p<0.05)$, no ocurriendo lo mismo con el resto de las dimensiones analizadas.

Por otro lado, para analizar la relación entre las puntuaciones de las dimensiones de la inteligencia emocional, empleamos el coeficiente de correlación de 
Pearson. Encontrándose que la dimensión Interpersonal correlaciona positivamente con el factor Intrapersonal $(r=.65 ; p<.01)$ y con la Adaptabilidad $(r=.38 ; p<.05)$.

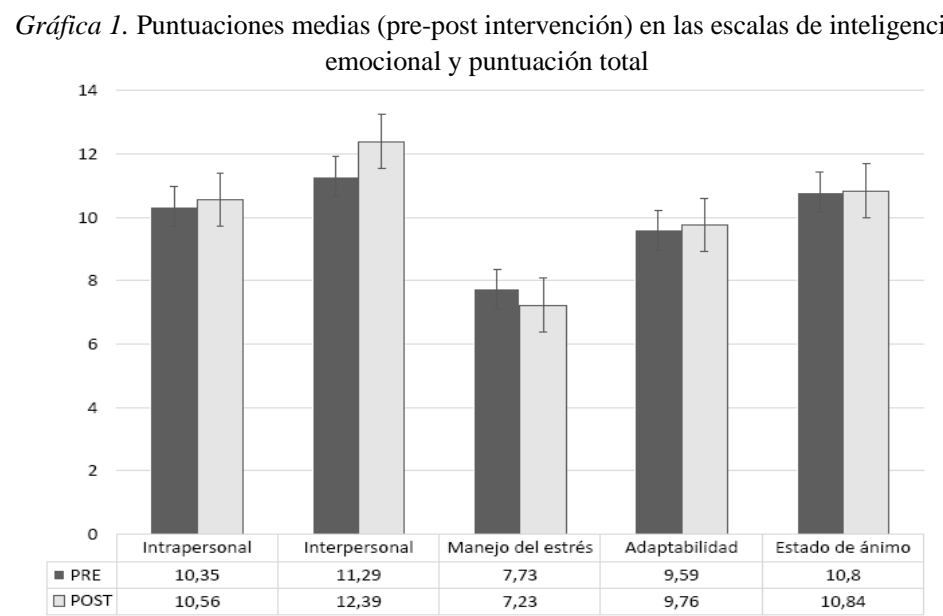

\section{DISCUSIÓN}

El programa de intervención que combina las técnicas de estimulación cognitiva con el entrenamiento de aspectos relacionados con la inteligencia emocional, potencia los efectos de una intervención basada exclusivamente en la mejora del desempeño cognitivo (Eich y Schooler, 2000), al contrario de lo que algunos autores refieren (Coats y Blanchard-Fields, 2008). De ahí que la aplicación del PECI-PM, mejore de forma generalizada de la mayoría de dimensiones que integran la inteligencia emocional, poniendo de manifiesto la posibilidad de educar las emociones a edades avanzadas (Scheibe y Carstensen, 2010; Scheibe y Zacher, 2013).

Por otro lado, el hecho de que la dimensión Manejo del Estrés sea la única en la que no se ha observado mejoría, nos lleva a replantear el peso de esta dimensión en el constructo inteligencia emocional a edades avanzadas (Birditt, 2013), tal y como ya se indicaba por los autores en el análisis de las propiedades psicométricas del instrumento utilizado (Pérez-Fuentes et al., 2014). Este tipo de resultados, permiten abrir de nuevo el debate sobre las diferencias de edad en cuanto a la forma de afrontar las situaciones estresantes (Márquez et al., 2006; Neupert et al., 2007). De igual modo, estos resultados estarían en la línea de estudios previos en los que la percepción de la autoeficacia del afrontamiento al estrés y su relación con las emociones, sigue siendo un tema de discusión (Kraaij et al., 2002). 
En cuanto a los cambios en el manejo del estrés con la edad, las propuestas más recientes pasan por la mediación de otros factores, como la percepción de autoeficacia o el apoyo social (Trouillet et al., 2009), muchos de ellos ya presentes en la adultez temprana (Brennan et al., 2012). Por tanto, no es posible afirmar que la capacidad para afrontar situaciones estresantes, pueda darse con características propias para el colectivo de mayores, aunque sería un aspecto a estudiar en profundidad en futuras investigaciones.

Finalmente, la existencia de una correlación significativa del factor Interpersonal con el Intrapersonal y la Adaptabilidad, revela el valor añadido de las relaciones sociales en la vejez, tanto en la configuración de la identidad personal y social del anciano (Catelli y Lanza, 2011), como en la capacidad de adaptación a los cambios propios de esta etapa (Bucks et al., 2008; Suzuki y Akiyama, 2013).

En definitiva, los resultados obtenidos, ponen de manifiesto el papel de la inteligencia emocional en la adquisición de recursos, para hacer frente a los cambios que acontecen en la vejez, lo que se traduce en una mejora de la calidad de vida (Pérez-Fuentes et al., 2012).

\section{Agradecimientos}

Este trabajo cuenta con la colaboración del Proyecto Almería Urban, cofinanciado por fondos FEDER y el Excmo. Ayuntamiento de Almería.

\section{REFERENCIAS}

Bar-On, R. (2004). The Bar-On Emotional Quotient Inventory (EQ-i): Rationale, description, and summary of psychometric properties. En G. Geher (Ed.), Measuring emotional intelligence: Common ground and controversy (pp. 111-142). Hauppauge, NY: Nova Science Publishers.

Bar-On, R. y Parker, J.D.A. (2000). Emotional Quotient Inventory: Youth Version (EQi:YV): Technical manual. Toronto, Canada: Multi-Health Systems.

Bernarás, E., Garaigordobil, M. y de las Cuevas, C. (2011). Inteligencia emocional y rasgos de personalidad. Influencia de la edad y el género durante la edad adulta y la vejez. Boletín de Psicología, 103, 75-88.

Birditt, K.S. (2013). Age differences in emotional reactions to daily negative social encounters. The Journals of Gerontology: Psychological Sciences, 69(4), 557-566. doi: 10.1093/geronb/gbt045

Brennan, P.L., Holland, J.M., Schutte, K.K. y Moos, R.H. (2012). Coping trajectories in later life: A 20 year predictive study. Aging and Mental Health, 16(3), 305-316. doi: 10.1080/13607863.2011.628975

Bucks, R.S., Garner, M., Tarrant, L., Bradley, B.P. y Mogg, K. (2008). Interpretation of emotionally ambiguous faces in older adults. The Journals of Gerontology Series B: Psychological Sciences and Social Sciences, 63(6), 337-343. 
Cabanach, R.G., Fariña, F., Freire, C., González, P. y Ferradás, M.M. (2013). Diferencias en el afrontamiento del estrés en estudiantes universitarios hombres y mujeres. European Journal of Education and Psychology, 6(1), 19-32. doi: 10.1989/ejep.v6i1.100

Castelli, L. y Lanza, F. (2011). Long life to emotions: Emotional response categorisation across the lifespan. Cognition \& Emotion, 25(8), 1520-1525. doi: 10.1080/02699931.2011.561551

Charles, S. y Carstensen, L.L. (2010). Social and emotional aging. Annual Review of Psychology, 61, 383. doi: 10.1146/annurev.psych.093008.100448

Charles, S.T. y Piazza, J.R. (2007). Memories of social interactions: Age differences in emotional intensity. Psychology and Aging, 22, 300-309.

Coats, A.H. y Blanchard-Fields, F. (2008). Emotion regulation in interpersonal problems: the role of cognitive-emotional complexity, emotion regulation goals, and expressivity. Psychology and Aging, 23(1), 39-51. doi: 10.1037/0882-7974.23.1.39

Coen, R.F., Flynn, B., Rigney, E., O'Connor, E., Fitzgerald, L., Murray, C., Dunleavy, C., McDonald, M., Delaney, D., Merriman, N. y Edgeworth, J. (2011). Efficacy of a cognitive stimulation therapy programme for people with dementia. Irish Journal of Psychological Medicine, 28(3), 145-147. doi: 10.1017/S0790966700012131

Diener, E. y Chan, M.Y. (2011). Happy people live longer: Subjective well-being contributes to health and longevity. Applied Psychology: Health and Well-Being, 3(1), 1-43. doi:10.1111/j.1758-0854.2010.01045.x

Downing, N., Smith, M.M., Beglinger, L.J., Mills, J., Duff, K., Rowe, K.C., Epping, E., Paulsen, J.S., PREDICTHD Investigators of the Huntington Study Group. (2012). Perceived stress in prodromal Huntington disease. Psychology and Health, 27(2), 196-209. doi: $10.1080 / 08870446.2010 .529141$

Eich, E. y Schooler, J.W. (2000). Cognition and Emotion Interactions. En E. Eich, J.F. Kihlstrom, G.H. Bower, J.P. Forgas y P.M. Niedenthal (Eds.), Cognition and Emotion (pp. 3-29). Nueva York: Oxford University Press.

Extremera, N. y Fernández-Berrocal, P. (2006). Emotional Intelligence as Predictor of Mental, Social, and Physical Health in University Students. The Spanish Journal of Psychology, 9, 45-51. doi: 10.1017/S1138741600005965

Kraaij, V., Garnefski, N. y Maes, S. (2002). The joint effects of stress, coping, and coping resources on depressive symptoms in the elderly. Anxiety, Stress \& Coping, 15(2), 163177. doi: $10.1080 / 10615800290028468$

Kunzmann, U. y Richter, D. (2009). Emotional reactivity across the adult life span: The cognitive pragmatics make a difference. Psychology and Aging, 24(4), 879. doi: $10.1037 / \mathrm{a} 0017347$

Lloyd, S.J., Malek-Ahmadi, M., Barclay, K., Fernández, M.R. y Stanley, M. (2012). Emotional intelligence (EI) as a predictor of depression status in older adults. Archives of Gerontology and Geriatrics, 55(3), 570-573. doi: 10.1016/j.archger.2012.06.004

Márquez, M., Montorio, I., Izal, M. y Losada, A. (2006). Predicción del nivel de ansiedad a partir de la intensidad emocional y el afrontamiento cognitivo en situaciones amenazantes en personas jóvenes y mayores. Ansiedad y Estrés, 12(2-3), 305-316.

Martin, M., Clare, L., Altgassen, A.M., Cameron, M.H. y Zehnder, F. (2011). Cognition-based interventions for healthy older people and people with mild cognitive impairment. Cochrane Database System Reviews, 1(1). doi: 10.1002/14651858.CD006220.pub2

Morales, F.M., Trianes, M.V. y Casado, A.M. (2013). Eficacia de un programa para fomentar la adquisición de competencias solidarias en estudiantes universitarios. European Journal of Education and Psychology, 6(2), 95-104. doi: 10.1989/ejep.v6i2.106 
Navarro, B., Latorre, J.M. y Ros, L. (2009). Inteligencia Emocional Autoinformada en la vejez, un estudio comparativo con el TMMS-24. En P. Fernandez-Berrocal (Ed.), Avances en el estudio de la Inteligencia Emocional (pp. 331-335). Madrid: Fundación Marcelino Botín.

Neupert, S.D., Almeida, D.M. y Charles, S.T. (2007). Age differences in reactivity to daily stressors: The role of personal control. The Journals of Gerontology Series B: Psychological Sciences and Social Sciences, 62(4), 216-225.

Orgeta, V. y Phillips, L.H. (2007). Effects of age and emotional intensity on the recognition of facial emotion. Experimental Aging Research, 34(1), 63-79. doi: $10.1080 / 03610730701762047$

Pearlin, L.I. (2010). The life course and the stress process: Some conceptual comparisons. Journal of Gerontology: Social Sciences, 65B, 207-215. doi: 10.1093/geronb/gbp106

Pérez-Fuentes, M.C., Gázquez, J.J., Mercader, I. y Molero, M.M. (2014). Brief Emotional Intelligence Inventory for Senior Citizens (EQ-i-M20). Psicothema, 26(4), 524-530. doi: 10.7334/psicothema2014.166

Pérez-Fuentes, M.C., Molero, M.M., Osorio, M.J. y Mercader, I. (2014). Propuesta de intervención cognitiva en personas mayores: Programa de Estimulación Cognitiva e Inteligencia Emocional para Mayores. International Journal of Developmental and Educational Psychology, 1(1), 477-488.

Pérez-Fuentes, M.C., Ruiz, B., Molero, M.M., Gázquez, J.J. y Fernández, M. (2012). Inteligencia emocional: estudio descriptivo en mayores. En M.C. Pérez-Fuentes y J.J. Gázquez (Coords.), Salud y envejecimiento. Un abordaje multidisciplinar de la demencia (pp. 165-172). Granada: Grupo Editorial Universitario.

Reijnders, J., van Heugten, C. y van Boxtel, M. (2013). Cognitive interventions in healthy older adults and people with mild cognitive impairment: a systematic review. Ageing Research Reviews, 12(1), 263-275. doi: 10.1016/j.arr.2012.07.003

Rinaldis, M., Pakenham, K. y Lynch, B.M. (2012). A structural model of the relationships among stress, coping, benefit finding and quality of life in persons diagnosed with colorectal cancer. Psychology and Health, 27(2), 159-177. doi: 10.1080/08870441003768047

Scheibe, S. y Carstensen, L.L. (2010). Emotional aging: recent findings and future trends. The Journals of Gerontology Series B: Psychological Sciences and Social Sciences, 65B(2), 135-144. doi: 10.1093/geronb/gbp132

Scheibe, S. y Zacher, H. (2013). A lifespan perspective on emotion regulation, stress, and wellbeing in the workplace. En P.L. Perrewé, J. Halbesleben y C.C. Rosen (Eds.), Research in occupational stress and well-being (pp. 163-193). Bingley, UK: Emerald.

Segerstrom, S.C. y O'Connor, D.B. (2012). Stress, health and illness: four challenges for the future. Psychology and Health, 27(2), 128-140. doi: 10.1080/08870446.2012.659516

Suzuki, A. y Akiyama, H. (2013). Cognitive aging explains age-related differences in face-based recognition of basic emotions except for anger and disgust. Aging, Neuropsychology and Cognition, 20(3), 253-270. doi: 10.1080/13825585.2012.692761

Tardif, S. y Simard, M. (2011). Cognitive stimulation programs in healthy elderly: a review. International Journal of Alzheimer's disease, 2011, 1-13. doi:10.4061/2011/378934

Teixeira, C.V.L., Gobbi, L.T.B., Corazza, D.I., Stella, F., Costa, J.L.R. y Gobbi, S. (2012). Nonpharmacological interventions on cognitive functions in older people with mild cognitive impairment (MCI). Archives of Gerontology and Geriatrics, 54(1), 175-180. doi: 10.1016/j.archger.2011.06.029

Trouillet, R., Gana, K., Lourel, M. y Fort, I. (2009). Predictive value of age for coping: the role of self-efficacy, social support satisfaction and perceived stress. Aging \& Mental Health, 13(3), 357-366. doi: 10.1080/13607860802626223 
Woods, B., Aguirre, E., Spector, A.E. y Orrell, M. (2012). Cognitive stimulation to improve cognitive functioning in people with dementia. Cochrane Database System Reviews, 2. doi: 10.1002/14651858.CD005562.pub2

$\mathrm{Xu}$, J. y Roberts, R.E. (2010). The power of positive emotions: It's a matter of life or death. Subjective well-being and longevity over 28 years in a general population. Health Psychology, 29(1), 9-19. doi: 10.1037/a0016767

Yesavage, J.A., Brink, T.L., Rose, T.L., Lum, O., Huang, V., Adey, M. y Leirer, V.O. (1983). Development and validation of geriatric depression screening scale: A preliminary report. Journal of Psychiatric Research, 17(1), 37-49.

Recibido: 25 de septiembre de 2014 Recepción Modificaciones: 13 de octubre de 2014 Aceptado: 19 de octubre de 2014 